\title{
Measurement of the radiation field at the Collider Detector at Fermilab
}

\author{
Kostas Kordas, Saverio D’Auria, Andy Hocker, Susan McGimpsey, Richard J. Tesarek, and Steven Worm
}

\begin{abstract}
We present direct measurements of the spatial distribution of both ionizing radiation and low energy neutrons $\left(E_{n}<200 \mathrm{keV}\right)$ inside the tracking volume of the Collider Detector at Fermilab (CDF). Using data from multiple exposures we are able to separate the contributions from beam losses and proton-antiproton collisions. Initial measurements of leakage currents in the CDF silicon detectors show patterns consistent with predictions based on our measurements.
\end{abstract}

Index Terms-Radiation measurement, ionizing radiation, nonionizing radiation, radiation field, radiation damage, radiation damage in silicon.

\section{INTRODUCTION}

$\mathbf{T}$ HE Collider Detector at Fermilab (CDF) [1] records the products of interactions between protons and antiprotons with a center of mass energy of $1.96 \mathrm{TeV}$. The trajectories of the interaction products are recorded by a series of semiconductor and gaseous detectors within $1.5 \mathrm{~m}$ of the interaction point. The characteristics of these devices are modified by large, chronic radiation exposures. Specifically, the leakage currents in the silicon-based detectors increase with the bulk damage of the silicon and eventually the detector ceases to provide useful data [2].

While a great deal of data are available on the damage of silicon devices, little is known about the radiation field induced by proton-antiproton collisions. Because semiconductor tracking detectors are expensive, both in time and effort, a detailed knowledge of the radiation field is needed in order to estimate the useful lifetime of a detector and plan for its replacement.

In this article we present a detailed measurement of the radiation field in the tracking volume of the CDF detector. We measure both ionizing radiation and low energy neutrons using

Manuscript received November 22, 2002. This work was supported by the U.S. Department of Energy and the National Science Foundation; the UK Particle Physics and Astronomy Research Council; and the Natural Sciences and Engineering Research Council of Canada.

Kostas Kordas is the corresponding author and is with the University of Toronto, Toronto, Ontario M5S 1A7, Canada (e-mail: kordas@fnal.gov)

Saverio D'Auria is with Glasgow University, Glasgow G12 8QQ, United Kingdom (e-mail: dauria @fnal.gov)

Andy Hocker is with the University of Rochester, Rochester, New York 14627, USA (e-mail: hocker@fnal.gov)

Susan McGimpsey and Richard J. Tesarek are with the Fermi National Accelerator Laboratory, Batavia, Illinois 60510, USA (e-mails: mcgimpsey@fnal.gov and tesarek@fnal.gov, respectively)

Steven Worm is with Rutgers University, Piscataway, New Jersey 08855 USA (e-mail: worm@fnal.gov)

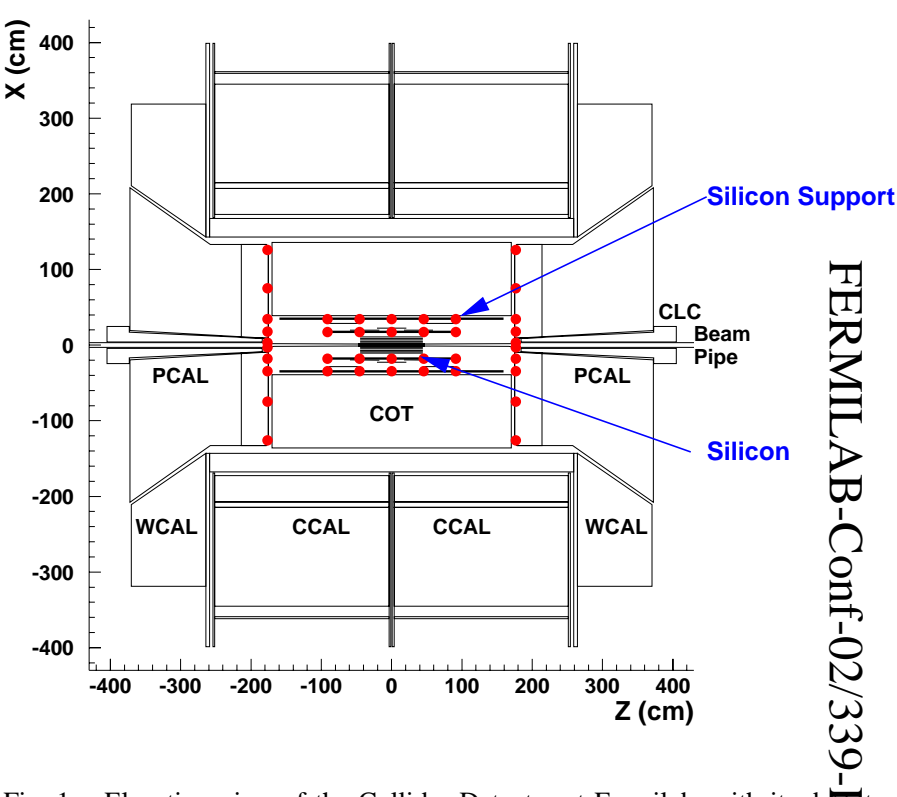

Fig. 1. Elevation view of the Collider Detector at Fermilab, with its detor component closest to the proton-antiproton collisions shown. The circles in the tracking volume denote the locations where we measure the radiation dose.

Thermal Luminescent Dosimeters (TLD's). From multiple posures, we are able to separate contributions to the radiation field due to collisions and protons lost from the beam. Fin we present a comparison of our field map with leakage current measurements in the silicon.

\section{RADIATION FIELD MEASUREMENT}

\section{A. The Collider Detector at Fermilab}

$\mathrm{CDF}$ records particles produced in proton-antiproton collisions by means of various detectors surrounding the beam line in a cylindrical geometry. Collisions of protons $(p)$ and antiprotons $(\bar{p})$ with an energy of $1.96 \mathrm{TeV}$ are provided by the Tevatron collider every $396 \mathrm{~ns}$. Protons travel along the $+z$ direction and collide with oncoming antiprotons at the heart of the CDF detector at $z=0$ (see Fig. 1). In the CDF cylindrical geometry we denote the distance from the beam line by $r$, and the azimuthal angle around the $z$-axis by $\phi$.

The silicon detectors are made of micro-strip sensors arranged in coaxial layers and are the first to measure charged particles produced at $p \bar{p}$ collisions. The inner layers (L00 


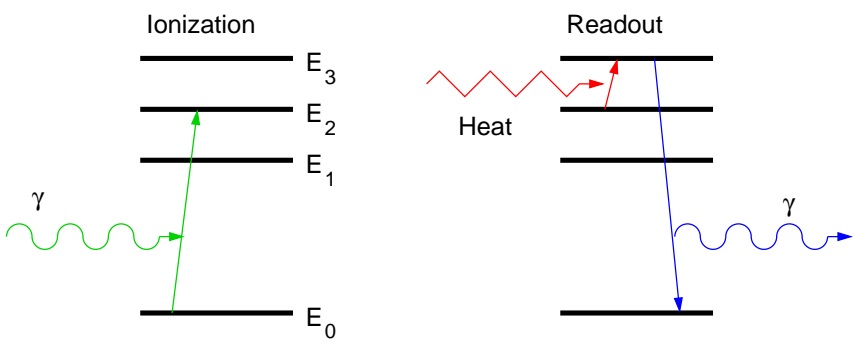

Fig. 2. The principle of thermal luminescence. Photon radiation brings the material in a meta-stable state, $E_{2}$, with a long lifetime (left). Heating the material leads to emission of visible photons (right).

and SVX) occupy the region $1.35 \mathrm{~cm}<r<10.6 \mathrm{~cm}$ and $-45 \mathrm{~cm}<z<+45 \mathrm{~cm}$; the outer layers (ISL) are situated in $20 \mathrm{~cm}<r<28 \mathrm{~cm}$, and $-95 \mathrm{~cm}<z<+95 \mathrm{~cm}$. The silicon detectors, along with the drift chamber (COT) at larger radii, are immersed in a $1.4 \mathrm{~T}$ solenoidal magnetic field. Outside the tracking volume, calorimeters (CCAL, PCAL, and WCAL in Fig. 1) measure the total energy of neutral and charged particles from the proton-antiproton collisions, and they are surrounded by muon detectors (not shown in the figure).

The number of $p \bar{p}$ collisions at the center of CDF is recorded by the Cherenkov Luminosity Counter (CLC) [3], by means of the Cherenkov radiation from charged particles produced at the proton-antiproton interactions. Further up-stream (not shown in Fig. 1), and on either side of the detector, two sets of scintillator counters surrounding the beam pipe record losses from protons and antiprotons ejected from the beam line. Proton(antiproton) losses are defined as the coincidence of a counter signal with a proton(antiproton) bunch crossing the plane of the scintillator on its way into the CDF detector.

\section{B. Thermal Luminescent Dosimeters}

Two types of Harshaw TLD chips are used for the radiation measurements. One type (TLD-700) is based on ${ }^{7} \mathrm{LiF}$ and is sensitive to ionizing radiation and photons. Ionizing radiation passing through the dosimeter brings the material in a metastable state with very long lifetime. Heating the TLD chip leads to a transition back to ground state accompanied by the emission of a photon (see Fig. 2). The number of photons produced is proportional to the population in these meta-stable states, which is in turn proportional to the amount of ionizing radiation that has traversed the TLD chip. The other dosimeter type (TLD-600) is based on ${ }^{6} \mathrm{LiF}$ and is sensitive to both ionizing radiation and low-energy neutrons $\left(E_{n}<200 \mathrm{keV}\right)$, as seen in Fig. 3. The reaction $n+{ }^{6} \mathrm{Li} \rightarrow{ }^{3} \mathrm{H}+\alpha$ results in a transition to the meta-stable state discussed above, by means of the recoiling tritium $\left({ }^{3} \mathrm{H}\right)$ and helium $(\alpha)$ nuclei.

Dosimeters are grouped in two triplets, one of each TLD type, and put in $3.18 \mathrm{~cm} \times 1.59 \mathrm{~cm}$ holders made of $0.79 \mathrm{~mm}$ thick FR-4 (see Fig. 4). The TLD's are held in place by $76 \mu \mathrm{m}$ thick kapton tape, and are subsequently placed in CDF to accumulate radiation.
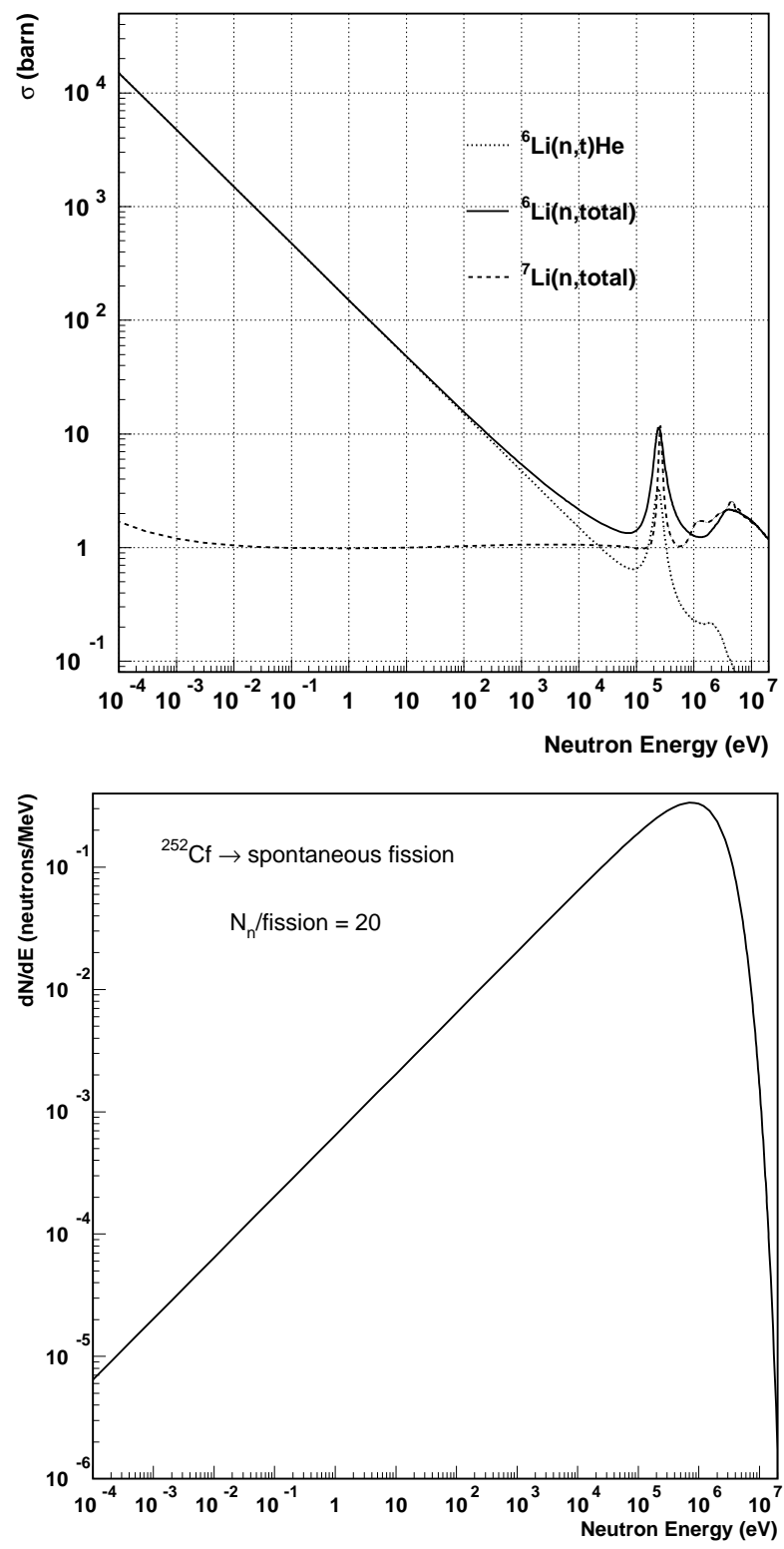

Fig. 3. Neutron absorption cross section as a function of neutron energy for ${ }^{6} \mathrm{Li}$ and ${ }^{7} \mathrm{Li}$ (top). Neutron energy spectrum from ${ }^{252} \mathrm{Cf}$ used to calibrate the neutron dose (bottom).

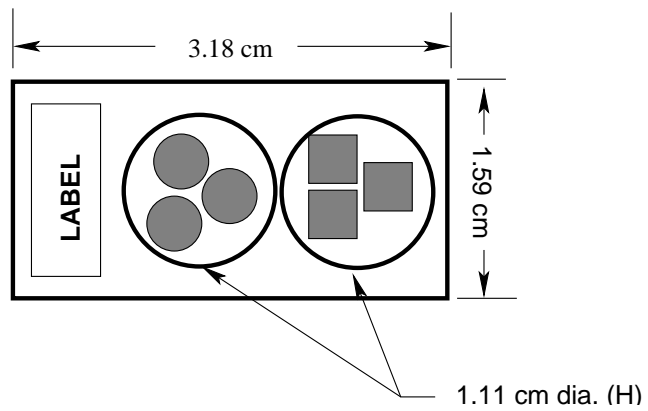

Fig. 4. A $0.79 \mathrm{~mm}$ thick FR-4 TLD holder. TLD-700 (round) and TLD-600 (square) dosimeters are kept in place by $76 \mu \mathrm{m}$ thick kapton tape. 

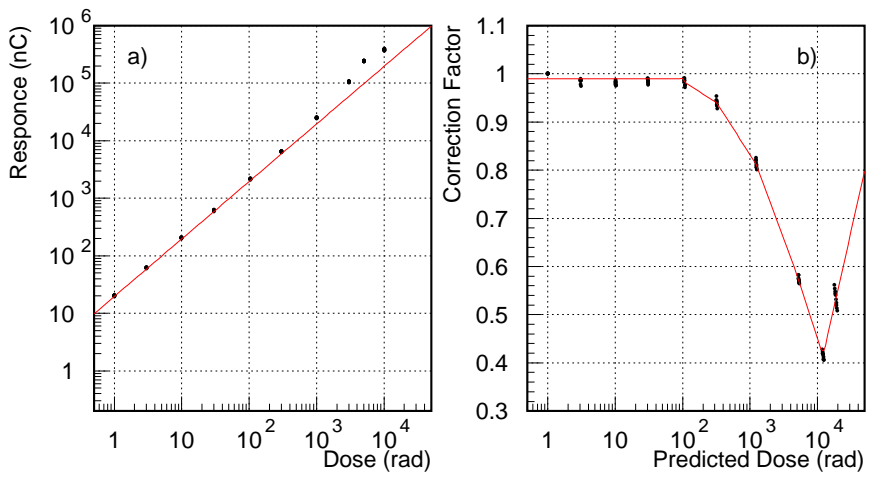

Fig. 5. a) Response of TLD-700 dosimeters to ionizing radiation as a function of received dose; note the super-linear behavior for doses above $100 \mathrm{rad}$. b) The non-linearity correction factor as a function of the dose estimated from the linear-response assumption.

\section{Calibration and Dosimetry}

We calibrate the TLD response to ionizing radiation with a $1 \mathrm{rad}$ photon exposure from a ${ }^{137} \mathrm{Cs}$ source [4]. A calibration factor (in $\mathrm{rad} / \mathrm{nC}$ ) for each TLD chip is then determined by heating up the chip and measuring the light yield using a Harshaw model 2000 TLD reader [5]. A reproducibility of $\sim 1 \%$ and a chip-to-chip variation of $\sim 3 \%$ is observed.

LiF TLD's are known to exhibit non-linearity for doses above $100 \mathrm{rad}$. In order to account for this behavior, we expose a small sample of TLD's to doses up to $10 \mathrm{krad}$ and we measure a correction factor, defined as the ratio of the received dose over the dose estimated from the linear-response assumption (see Fig. 5).

The response of the TLD-600 chips to neutrons is calibrated with a $1 \mathrm{mrad}$ exposure to a ${ }^{252} \mathrm{Cf}$ source (see Fig. 3 ). We obtain $\mathrm{a} \sim 10 \%$ reproducability and $\mathrm{a} \sim 15 \%$ chip-to-chip variation.

We extract the ionizing radiation, $D_{\gamma}(\mathrm{rad})$, each TLD-700 chip has received due to its exposure in the detector volume, by using the expression:

$$
D_{\gamma}=C_{\gamma, 7} k_{\gamma, 7} R_{7}-D_{\gamma, c t r l}
$$

where $R_{7}$ is the reading (nC) from this TLD chip, $k_{\gamma, 7}$ is the calibration factor $(\mathrm{rad} / \mathrm{nC})$ for its response to ionizing radiation, $C_{\gamma, 7}$ is the non-linearity correction factor, and $D_{\gamma, c t r l}$ is the background ionizing radiation dose measured by a number of control TLD-700 chips which were not placed in the detector.

Averaging the doses measured by the three TLD chips in a given holder, we obtain the ionizing radiation dose, $D_{\gamma}$, at the location of the TLD holder in study. We then calculate the neutron dose, $D_{n}$ (rad), from the TLD-600 chips in that holder, as follows:

$$
D_{n}=\frac{k_{n, 6}}{k_{\gamma, 6}}\left(C_{\gamma, 6} k_{\gamma, 6} R_{6}-D_{\gamma}\right)-D_{n, c t r l},
$$

where $R_{6}$ is their reading (nC), $k_{\gamma, 6}$ and $k_{n, 6}$ are the appropriate calibration factors, $C_{\gamma, 6}$ is the non-linearity correction factor, and $D_{n, c t r l}$ is the background neutron dose measured by a sample of control TLD-600 dosimeters.
TABLE I

BEAM CONDITIONS AT CDF FOR THE TWO TLD EXPOSURE PERIODS

\begin{tabular}{c|cc|cc|c}
\hline & \multicolumn{2}{|c|}{ Beam $\left(\times 10^{19}\right)$} & \multicolumn{2}{|c|}{ Losses $\left(\times 10^{9}\right)$} & $\int L d t$ \\
Period & $p$ & $\bar{p}$ & $p$ & $\bar{p}$ & $\left(\mathrm{pb}^{-1}\right)$ \\
\hline Feb. - May 2001 & 0.0703 & 0.0082 & 15.3 & 2.02 & 0.058 \\
May - Oct. 2001 & 1.56 & 0.137 & 40.9 & 10.2 & 10.7 \\
\hline
\end{tabular}
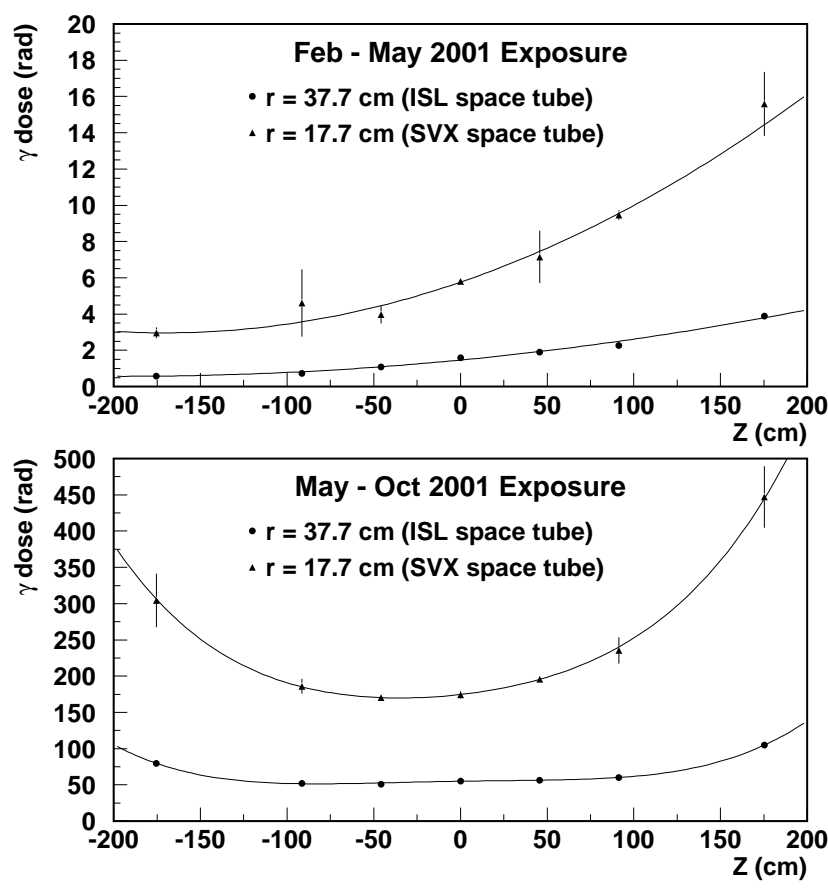

Fig. 6. Ionizing radiation dose as a function of $z$ for the two exposure periods. Top: the pattern during the first exposure period, dominated by proton beam losses (proton direction is $+z$ ). Bottom: the dose during the second exposure period, dominated by proton-antiproton collisions around $z=0$.

\section{The radiation measurements}

TLD measurements are taken during two different periods of the Tevatron operations, in three regions in the CDF tracking volume (Fig. 1): i) on the cylinder supporting the inner silicon (SVX), at 25 locations ( 5 along the $z$-axis and 5 in $\phi$ ), at $r=17.7 \mathrm{~cm}$, ii) on the support structure of the outer silicon (ISL), at 40 locations ( 5 along the $z$-axis and 8 in $\phi$ ), at $r=$ $37.7 \mathrm{~cm}$, and iii) on the inner faces of the plug calorimeter (PCAL), at 80 locations ( 5 in $r$ and 8 in $\phi$ ), at $z= \pm 175.3 \mathrm{~cm}$. Table I shows the number of protons and antiprotons in the Tevatron, the number of lost beam particles recorded, and the number of collisions during the two exposure periods. From February to May 2001 the ratio of proton losses to collisions was $264 \times 10^{9} / \mathrm{pb}^{-1}$, whereas from May to October 2001 collisions were dominant and the ratio was $3.9 \times 10^{9} / \mathrm{pb}^{-1}$. The Tevatron is currently operating with $\sim 2 \times 10^{9}$ loss counts per $\mathrm{pb}^{-1}$ of collisions.

The ionizing radiation during these two periods is shown in Fig. 6. Each point on the plots is the average of the various measurements in $\phi$ for the given $z$ location. The uncertainty shown is the RMS spread of these measurements. Collisions 

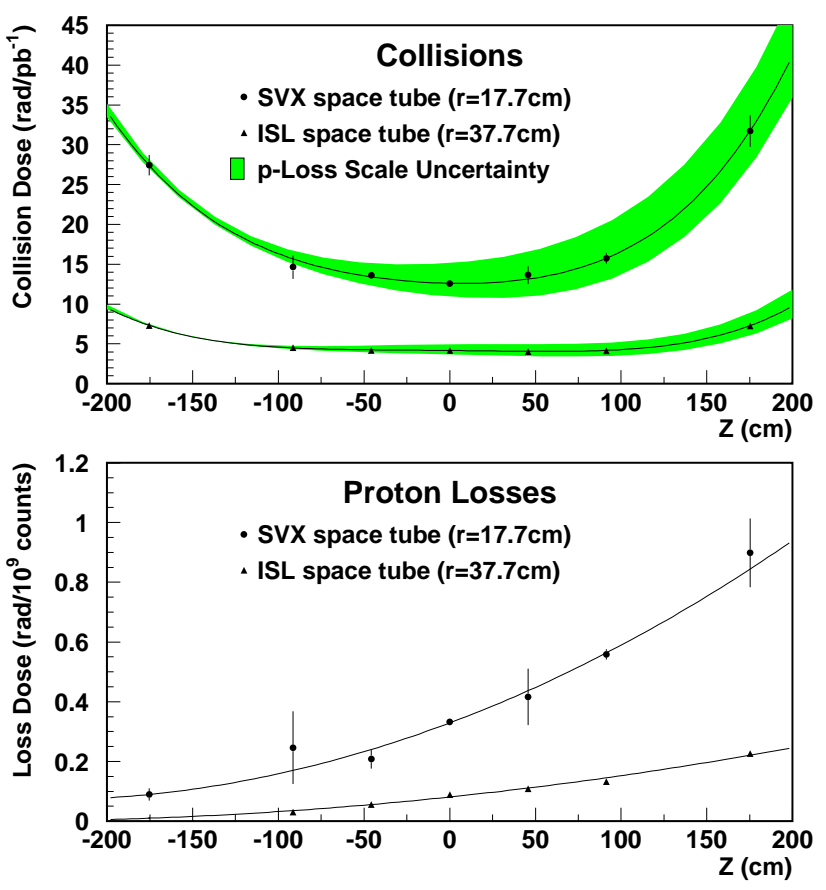

Fig. 7. Ionizing radiation dose, as a function of $z$, due to $p \bar{p}$ collisions (top), and due to beam losses (bottom). The shaded band represents the systematic uncertainty from the beam loss measurements. For the second exposure period, the ratio of proton losses to collisions was $3.9 \times 10^{9}$ counts $/ \mathrm{pb}^{-1}$.

result in a roughly symmetric distribution around the nominal $p \bar{p}$ collision point $(z=0)$, whereas losses yield asymmetric distributions.

Assuming that the radiation at a given point is the linear super-position of contributions from losses and collisions, we solve for these two components during the two exposure periods. The resulting ionizing radiation fields are shown in Fig. 7. From them, we estimate that during the second exposure period, $p \bar{p}$ collisions contribute a dose of $\sim 13 \mathrm{rad}$ per $\mathrm{pb}^{-1}$ at a radius of $37.7 \mathrm{~cm}$ at $z=0$. The corresponding dose from losses is $\sim 0.3 \mathrm{rad}$ per $10^{9}$ counts $\simeq 1.2 \mathrm{rad}$ per $3.9 \times 10^{9}$ counts $\simeq 1.2 \mathrm{rad}$ per $\mathrm{pb}^{-1}$ of collisions. This is a contribution of $\sim 8 \%$ to the ionizing radiation.

The neutron radiation doses for the two exposure periods are shown in Fig. 8. No $z$-dependence in the region of the silicon detectors and no evident radial dependence of the neutron radiation is seen, in contrast to the ionizing radiation measurements.

\section{MODELING THE RADIATION FIELD}

The ionizing radiation measurements are parameterized using a model based on previous CDF measurements of the silicon radiation damage profile [6]. This model assumes cylindrical symmetry of the radiation around the beam line, with a radial dependence which follows a power law in $1 / r$, where $r$ is the distance from the beam line. For any point $(x, y)$ on a plane perpendicular to the beam axis at $z$, we write for the dose,
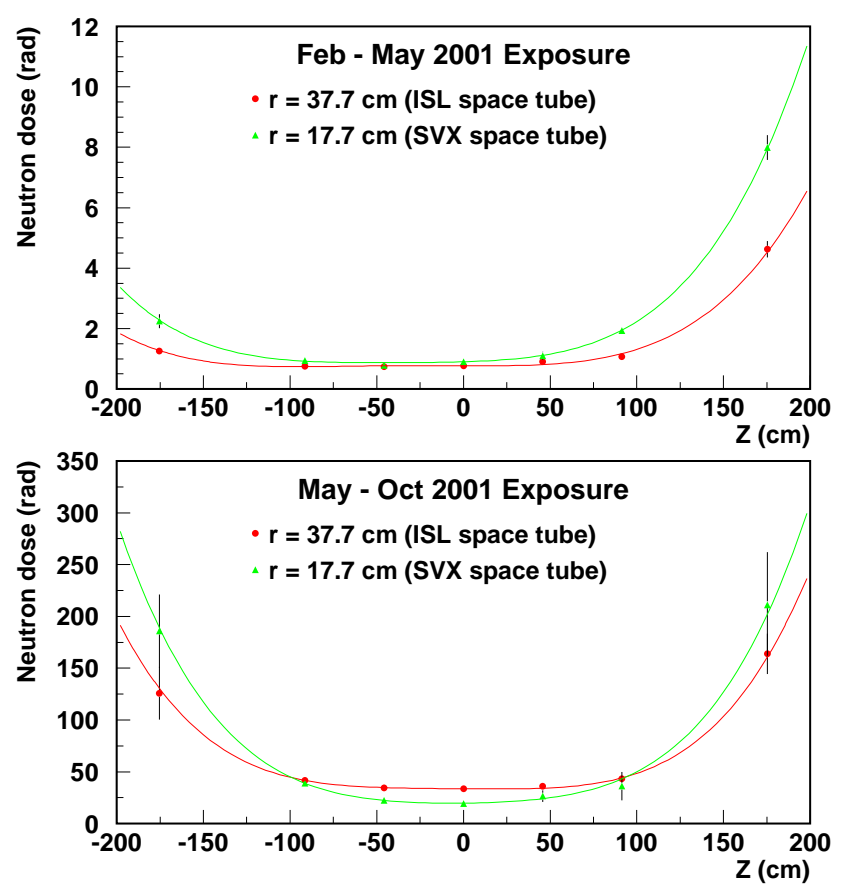

Fig. 8. Neutron radiation dose as a function of $z$ for the two exposure periods Top: the pattern during the first exposure period, dominated by proton beam losses (proton direction is $+z$ ). Bottom: the dose during the second exposure period, dominated by proton-antiproton collisions around $z=0$.
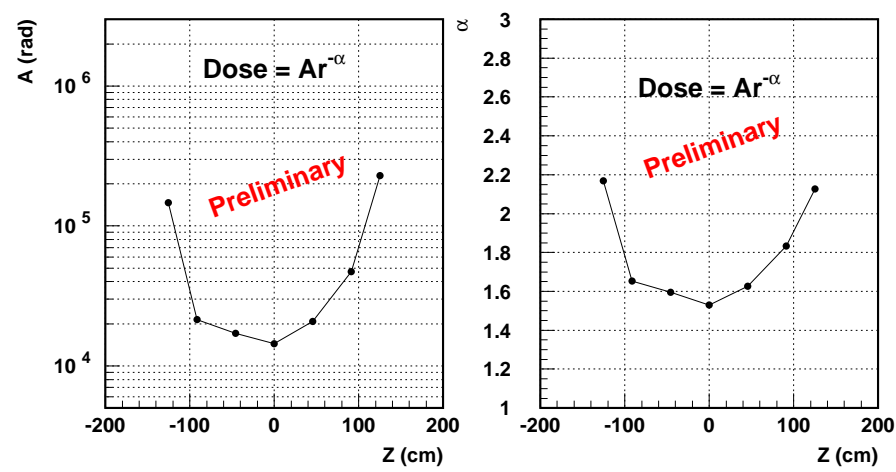

Fig. 9. Fit parameters of the radiation field model in Equation 3; normalization (left) and power law exponent (right) as a function of $z$.

$D(x, y)$ :

$$
D(x, y)=A\left(\frac{1}{\sqrt{\left(x-x_{0}\right)^{2}+\left(y-y_{0}\right)^{2}}}\right)^{\alpha},
$$

where $A$ is the absolute normalization, $\alpha$ is the power law exponent, and $\left(x_{0}, y_{0}\right)$ is the offset of the beam line compared to the detector $z$-axis. The normalization and the exponent exhibit a strong $z$-dependence, as seen in Fig. 9. For the region of the silicon $(-95 \mathrm{~cm}<z<+95 \mathrm{~cm})$, the power law exponent has values from $\alpha=1.5$ at $z=0$ to $\sim 1.8$ at $z=95 \mathrm{~cm}$.

Using our TLD measurements and this model, we quote that at a radius $r=3 \mathrm{~cm}$ on the plane $z=0$, we expect a dose of $300 \pm 60 \mathrm{rad}^{\mathrm{p} e r} \mathrm{pb}^{-1}$ of collisions. This number can be then 
used in conjunction with the power law of Equation 3, with $\alpha$ values from Fig. 9, to get the prediction of the radiation level at any point of our tracking volume.

\section{THE SILICON AS A RADIATION MONITOR}

The prediction of the radiation field reconstructed from the TLD measurements and the simple model presented above, can be tested by comparing to the radiation seen by the silicon detectors located closest to the beam line.

The increase in the detector leakage current $\left(\Delta I_{\text {leak }}\right)$ is related to the particle fluence, $\Phi$ (number of particles per unit area), through the relation:

$$
\Delta I_{\text {leak }}=\alpha_{\text {damage }} \cdot \Phi \cdot \mathrm{V},
$$

where $\alpha_{\text {damage }}$ is the damage factor which depends on the type of radiation traversing the silicon and on the temperature of the silicon. $V$ is the active volume of the silicon sensors. For minimum ionizing particles going through silicon kept at a temperature of $20^{\circ} \mathrm{C}$, we use $\alpha_{\text {damage }}=3 \times 10^{-17} \mathrm{~A} / \mathrm{cm} \mathrm{[7]}$. After correcting for the actual temperature of the silicon during the measurement period $\left(\sim 8^{\circ} \mathrm{C}\right)$, we solve Equation 4 for the fluence of particles through the innermost silicon layers, at $r \simeq 1.7 \mathrm{~cm}$.

The particle fluence calculated this way can be directly compared to the expected fluence given by the radiation field model discussed above. We convert the radiation dose to particle fluence by using a factor of $3.87 \times 10^{7}$ minimum ionizing particles per rad.

The measurements of the leakage currents in the silicon were performed during a later period than the TLD measurements. The particle flux (i.e., fluence per $\mathrm{pb}^{-1}$ ) is shown in Fig. 10 as a function of the azimuthal angle $\phi$ around the beam line. Notice that the pattern is not uniform because the beam line does not travel at the center of the beam pipe; it is offset by $-1.8 \mathrm{~mm}$ in $x$ and $4 \mathrm{~mm}$ in $y$ compared to the $z$-axis of cylindrical symmetry for the silicon detector. The points represent the flux resulting from the measured increase in the leakage currents at the innermost silicon layers. The curve is not a fit but a prediction using the model to extrapolate from the TLD measurements to the radial location of the silicon. It should be noted that the extrapolation is over an order of magnitude in $1 / r$. Despite the extrapolation, the two results agree to within $10 \%$.

\section{CONCLUSION}

Using Thermal Luminescent Dosimeters (TLD's) we have made a detailed measurement of the radiation field from ionizing radiation and low-energy neutrons $\left(E_{n}<200 \mathrm{keV}\right)$ in the tracking volume of the Collider Detector at Fermilab (CDF). Using data from two exposures we are able to separate the radiation field into components from proton beam losses and proton-antiproton collisions. This allows us to estimate the radiation field given any beam conditions.

When a simple model assuming a power law in $1 / r$ is used, fits to the data yield exponents with a strong $z$-dependence;

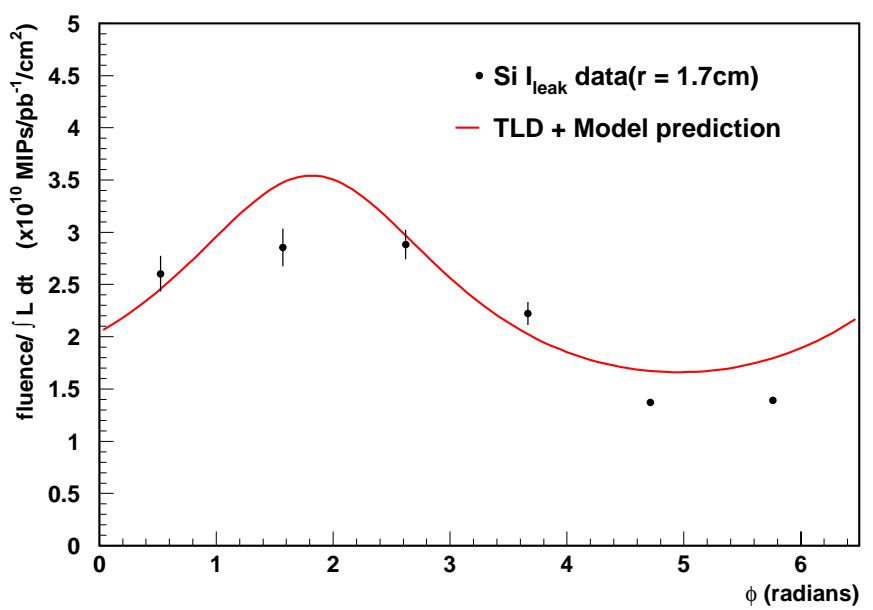

Fig. 10. Fluence (particles per unit area) per $\mathrm{pb}^{-1}$ of delivered luminosity, as a function of the azimuthal angle around the beam line. The points are from the leakage current measurements at the innermost silicon layer. The curve is the prediction of the radiation field model constructed from the TLD measurements.

$1.5-2.1$ in the CDF tracking volume. The TLD measurements predict a dose of $300 \pm 60 \mathrm{rad}^{\mathrm{per}} \mathrm{pb}^{-1}$ of collisions, at a distance $r=3 \mathrm{~cm}$ from the beam line, at $z=0$.

We find our TLD measurements in good agreement with leakage current measurements in the silicon.

We believe that our data can serve as a calibration point for simulations of the radiation environment in future hadron colliders.

\section{ACKNOWLEDGMENT}

The authors would like to thank the people at Fermilab's Radiation Physics Calibration Facility, the Silicon Detector Lab and CDF for their help in calibrating the TLD's, placing them to the holders and installing them in CDF.

\section{REFERENCES}

[1] R. Blair et al., The CDF II detector Technical Design Report, preprint FERMILAB-Pub-96/390-E, October 1996, unpublished.

[2] C. Leroy, S. Bates, B. Dezillie, M. Glaser, F. Lemeilleur and I. Trigger, "Study of charge collection and noise in non-irradiated and irradiated silicon detectors", Nucl. Instr. Meth., vol. A388, pp 289-296, 1997; F. Lemeilleur, M. Glaser, E. H. Heijne, P. Jarron and E. Occelli, "Neutron induced radiation damage in silicon detectors", IEEE Trans. Nucl. Sci., vol. 39, pp. 551-557, 1992.

[3] D. Acosta et al., "The CDF Cherenkov luminosity monitor", Nucl. Instr. and Meth., vol. A461, pp. 540-544, 2001

[4] F. Krueger and S. Hawke, "Calibration and On-Axis Characterization of the Source Projector Facility at the Radiation Physics Calibration Facility", Fermilab Radiation Physics internal note 121, January 1996, unpublished.

[5] Harshaw Chemical Company, Harshaw model 2000 TLD reader: Thermo RMP, 6801, Cochran Road, Solon, OH 44139, USA, 2000. The model 2000 TLD reader used for these measurements at Fermilab was modified to extend the reading cycle to 1 second.

[6] D. Amidei et al., "The silicon vertex detector of the Collider Detector at Fermilab", Nucl. Instr. and Meth., vol. A350, pp. 73-130, 1994.

[7] K. Hagiwara et al., "Review of particle physics", Phys. Rev. D, vol. 66, no. 1-I, p. 010001-213, 2002. 